\title{
Carotid artery intra-plaque attenuation variability using computed tomography
}

(1) CrossMark

Luca Saba ${ }^{{ }^{*}}$ (D), Michele Anzidei ${ }^{2}$, Carlo Nicola de $\mathrm{Cecco}^{3}$, Michele Porcu', Antonella Balestrieri ${ }^{1}$, Roberto Sanfilippo ${ }^{4}$, Marco Francone ${ }^{2}$, Alessio Mereu ${ }^{1}$, Pierleone Lucatelli ${ }^{2}$, Roberto Montisci ${ }^{4}$, Jasjit S. Suri ${ }^{5}$ and Max Wintermark ${ }^{6}$

\begin{abstract}
Background: In the CT assessment of the carotid plaque, the analysis of the attenuation value is a fundamental parameter in order to classify the type of the tissue that composes the plaque. The purpose of this paper is to assess the intra-plaque attenuation variability in order to verify the potential reproducibility of HU measurements.

Methods: In this retrospective study, 68 consecutive patients (males 42; average age $64 \pm 11$ years) that underwent $\mathrm{CT}$ of carotid arteries were included. Exams were performed before and after administration of contrast medium and in each slice 4 different circular or elliptical ROls ( $\geq 1$ and $\leq 2 \mathrm{~mm}^{2}$ ) were traced and attenuation values were recorded. Wilcoxon and Mann-Whitney test were used to test the differences between the ROI.

Results: A total of 192 slices were analysed. The average value of attenuation before contrast medium was 41 . 591 (SD 8.1) HU and 54.159 (SD 15.7) in post-contrast scan. Mann-Whitney test did not find statistically significant difference among the ROI in the pre-contrast scan whereas a statistically significant differences was found in post-contrast scan. Wilcoxon analysis showed a statistically significant difference $(p$ value $=0.001)$ between pre and post-contrast attenuation.

Conclusion: In conclusion, results of our study suggest that ROI sampling performed in the $\mathrm{CT}$ dataset acquired after administration of contrast medium show significant degree of heterogeneity and a statistically significant differences compared to the baseline measurement. This effect may be driven by a different amount of contrast acquisition in some areas of the carotid artery plaque.
\end{abstract}

\section{Background}

Cerebrovascular ischemic events are the third leading cause of death after acute myocardial infarction and cancer and represent the second cause of disability in the Western world [1, 2]. A significant portion of the ischemic events have their cause in the embolization occurred from the carotid artery $[3,4]$.

In the past years it was thought that the stenosis degree was the leading parameter related to the patient's risk to develop cerebrovascular events [5] but in the last decade several researches have demonstrated that the plaque morphology and characteristics play a fundamental role in the development of embolic cascade [6, 7]. Therefore identification of carotid artery features that are associated with plaque vulnerability is extremely

\footnotetext{
* Correspondence: lucasaba@tiscali.it

${ }^{1}$ Department of Radiology, Azienda Ospedaliero Universitaria (A.O.U.), di Cagliari - Polo di Monserrato, s.s. 554 Monserrato, Cagliari 09045, Italy

Full list of author information is available at the end of the article
}

important in order to correctly stratify the risk of occurrence of cerebrovascular events $[8,9]$ that's why several imaging techniques are trying to identify those characteristics $[10,11]$.

Ultrasonography (US) is nowadays considered the first-line exam [12] but in most of centres Magnetic Resonance Imaging (MRI) or Computed Tomography (CT) are required to confirm or to rule out the presence of pathological stenosis and $\backslash$ or plaque's characteristics related to the vulnerability $[13,14]$. MRI offers, other than the potentiality of quantify the degree of stenosis, the opportunity to identify the presence of intra-plaquehemorrhage (IPH), lipid-rich necrotic core (LRNC) as well as the status of the fibrous cap $[15,16]$.

In the assessment of the carotid arteries, CT is a frequently used technique because of the wide availability, the rapid time execution and its potentiality to identify some features related to the plaque vulnerability [17-19]. In the $\mathrm{CT}$ assessment of the carotid plaque the analysis 
of the attenuation value is a fundamental parameter in order to classify the type of the tissue that composes the plaque [20].

The purpose of this paper is to assess the intra-plaque attenuation variability in order to verify the potential reproducibility of $\mathrm{HU}$ measurements.

\section{Methods}

\section{Study design and patient population}

In this retrospective study, 68 consecutive patients (males 42 , females 16; median age $64 \pm 11$ years, age range $44-82$ years) that underwent CTA between January 2012 and May 2012 were included. The IRB approval was obtained and because of the retrospective nature of the analysis patient's consent was waived.

In our Hospital CTA is performed after ultrasonography that is used as exam to rule-out significant atherosclerotic disease of the supra-aortic vessels. In particular CTA is performed when a) carotid sonography shows a pathological stenosis $(>50 \%$ according to the NASCET criteria [21]) or plaque's features related to plaque vulnerability (such as ulcers of irregular luminal morphology) b) sonography cannot adequately assess degree of stenosis and plaque's characteristics because of anatomical conditions.

\section{CTA technique}

None of the patients included in this research had any contraindications to iodinated contrast media. CTA was performed using a 16-multi-detector row CT system (Philips Brilliance, Eindhoven, Netherlands). Acquisitions were performed from the aortic arch to the circle of Willis before and after administration of contrast medium. Bolus tracking technique was used in all cases. The ROI trigger threshold was placed into the aortic arch and six seconds after the beginning of the i.v. administration of $80 \mathrm{ml}$ of pre-warmed contrast medium (Ultravist 370; Schering, Berlin, Germany) into median cubital vein (flow rate of 5 $\mathrm{ml} / \mathrm{s}$ ) the monitor scanning began. The trigger threshold was set at $+80 \mathrm{HU}$ above the baseline. Four seconds after having reached the threshold the angiographic phase began. CT technical parameters were: matrix $512 \times 512$, field of view 14-19 cm; slice thickness $0.6 \mathrm{~mm}$, interval $0.3 \mathrm{~mm}, 180-220 \mathrm{mAs} ; 120-140 \mathrm{kV}$. C-filter algorithm of reconstruction was applied.

\section{Plaque analysis}

The window parameters (center/level) was set at W850:L300 according to Saba et al [21] and in the first phase the observer (XX, 12 years of experience in vascular imaging) analysed the dataset acquired after administration of contrast material. The carotid artery plaque was identified and for each slice 4 different circular or elliptical ROIs were traced. The 4 ROI were exactly the same in each slice and their dimension was variable $\geq 1$ and $\leq 2 \mathrm{~mm}^{2}$ in the different slices. When small cluster of calcium were identified, these are avoided in the ROI tracings. We considered 2 main exclusion criteria 1) calcified plaques 2) presence of artefacts (movement or dental artefacts). In the second phase the basal scan were assessed. The observers identified the same slice correspondent to the angiographic phase and the same ROIs (for locations and dimension) used in the angiographic phase were applied in the slices (Fig. 1).

At the end of the analysis the ROI were grouped according to their $\mathrm{HU}$ value from the lower to the higher values and ROI 1 represents the lower $\mathrm{HU}$ values whereas ROI 4 the higher.

\section{Statistical analysis}

In this study the normality of each continuous variable group was tested using the Kolmogorov-Smirnov $Z$ test and appropriate tests for Gaussian or non-Gaussian values were selected. For Gaussian values, continuous data were described as the mean value \pm SD whereas for non-gaussian values median values were given. Wilcoxon test was applied to test the difference of attenuation of the ROIs traced before and after administration of contrast material whereas the Mann-Whitney test was used to test the differences between the ROI. A $p$ value $<0.05$ was regarded to indicate statistical significance association and all values were calculated using a two-tailed significance level. $R$ software (www.r-project.org) was employed for statistical analyses.

\section{Results}

Of the 136 carotid arteries that were imaged with CTA, sixteen carotid arteries were excluded because no evidence of plaque was found. Other 41 carotid arteries were excluded because their plaque was heavily calcified and other 12 because of the presence of artefacts (movements $n=6$ and dental artefacts $n=6$ ). The remaining 67 carotid artery plaques were analysed and 192 slices were found where it was possible to trace 4 ROIs with area between 1 and $2 \mathrm{~mm}^{2}$.

In the Table 1 are summarized the results of the attenuation values of the four ROIs in the basal and postcontrast phase. The average value of attenuation before contrast medium was 41.591 HU (SD 8.1) and 54.159 (SD 15.7) after its administration. The average value of the ROI used was $1.532 \mathrm{~mm}^{2}$ (SD 0.163).

By comparing the attenuation values among the four different ROIs (summarized in the Table 2) we found that in the basal scan no statistic differences among the four ROIs was found whereas in the scan performed after contrast material in most of cases there were difference among the ROI values. Standard deviation analysis was also performed (summarized in the Table 3). In the Fig. 2 is given a box-plot that shows the attenuation 


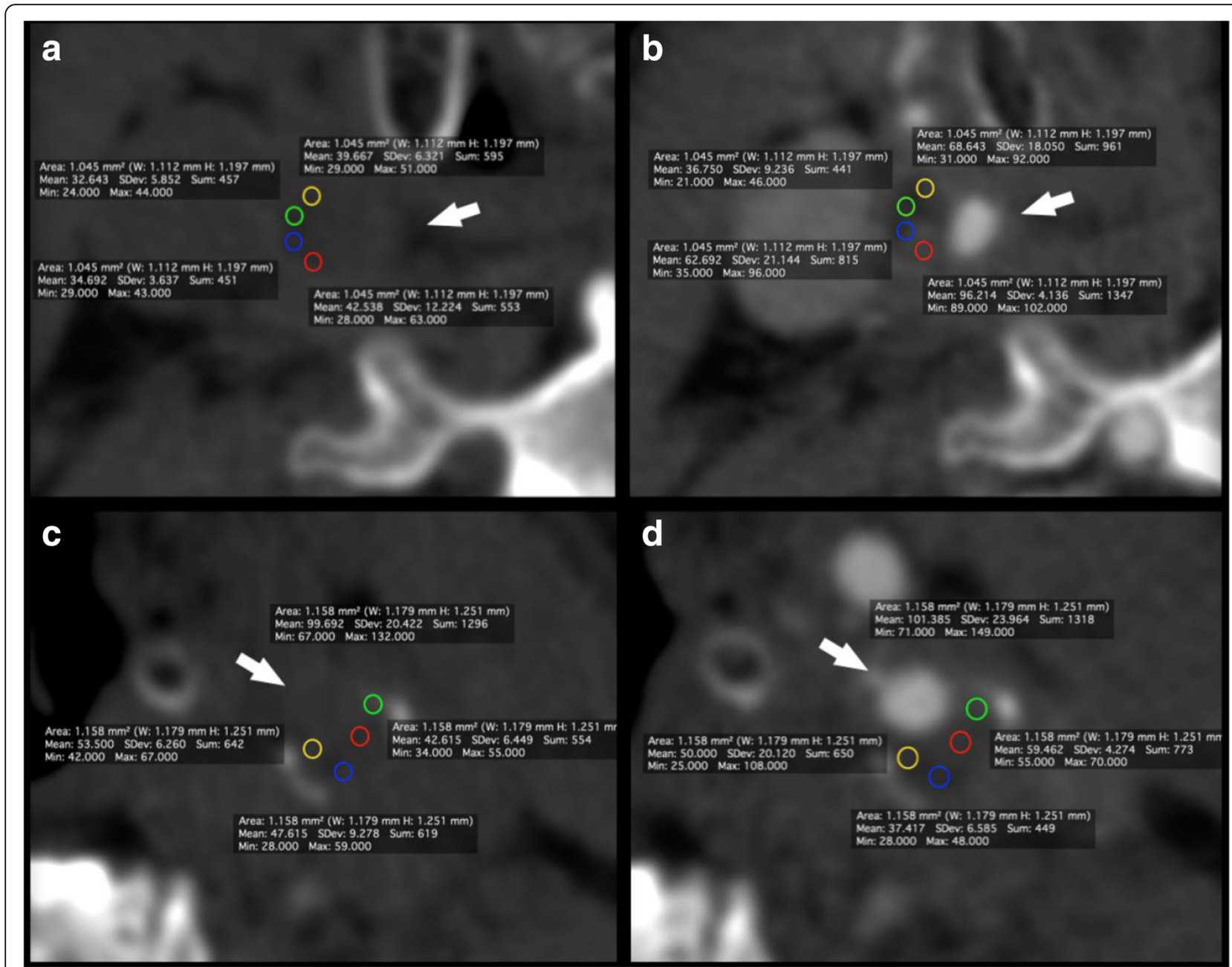

Fig. 1 In panel $\mathbf{a}$ and $\mathbf{b}$ the right carotid plaque of a 61 years old male patient is given. The 4 ROI are visible in the pre contrast (a) and post-contrast scan (b). In panel $\mathbf{c}$ and $\mathbf{d}$ the left carotid plaque of a 68 years old female patient is given. The $4 \mathrm{ROI}$ are visible in the pre contrast (c) and post-contrast scan (d). The white arrow indicates the lumen of the carotid artery

values of the ROI and the Standard Deviations before and after administration of contrast material.

Wilcoxon analysis was used to assess the difference of attenuation before and after administration of contrast material. By comparing the overall attenuation of the ROI of the plaques before and after administration of contrast medium we found a statistically significant difference $(p$ value $=0.001)$.

\section{Discussion}

Attenuation analysis of carotid artery plaque represents an important criterion for the identification of plaque's vulnerability because some types of tissue (fatty ones) are associated with an increased risk [22] of cerebrovascular events whereas others (calcified tissues) are associated with a reduced risk [23]. In this study our purpose was to assess the intra-plaque attenuation variability in order to verify the potential reproducibility of
HU measurements and to assess the polymorphism of the plaque.

Our analysis relies to previous histopathological studies that have been demonstrated that multiple type of tissues are present in the carotid artery plaques [24, 25] and that this features can be visible also in the CT images.

We excluded from the ROI tracings those areas where small calcium deposits or cluster of calcium were visible and we considered as exclusion criteria for a slice the presence of heavily calcified plaques (that determines $\mathrm{HU}$ values usually very high up to $800-1000$ ). Nowadays it is extremely important to obtain information that allows to identify and distinguish the fatty plaque (components) from the mixed plaques (components).

Moreover the structure of the carotid artery plaque, when big calcification are present, is characterized by the peripheral location of the calcium components with the 
Table 1 Summary values of attenuation (measured in $\mathrm{HU}$ ) of the ROls before and after administration of contrast medium (with the Standard Deviation)

\begin{tabular}{|c|c|c|c|c|c|c|}
\hline & Mean & SD & Minimum & Maximum & $2.5-97.5 P$ & Normal Distr. \\
\hline ROI 1 basal & 33.818 & 11.0074 & 14 & 49 & $14.000-49.000$ & 0.3899 \\
\hline ROI 1 contrast & 40.273 & 9.1879 & 24 & 51 & $24.000-51.000$ & 0.6966 \\
\hline ROI 2 basal & 41.364 & 7.5534 & 28 & 52 & $28.000-52.000$ & 0.5753 \\
\hline ROI 2 contrast & 51.273 & 10.2868 & 31 & 62 & $31.000-62.000$ & 0.0512 \\
\hline ROI 3 basal & 41.909 & 11.5884 & 27 & 57 & $27.000-57.000$ & 0.0872 \\
\hline ROI 3 contrast & 56.273 & 13.8859 & 31 & 72 & $31.000-72.000$ & 0.3031 \\
\hline ROI 4 basal & 49.273 & 20.7079 & 28 & 99 & 28.000-99.000 & 0.0254 \\
\hline ROI 4 contrast & 68.818 & 19.0778 & 43 & 101 & $43.00-101.000$ & 0.8457 \\
\hline SD ROI 1 basal & 7.545 & 2.1616 & 5 & 11 & $5.000-11.000$ & 0.5274 \\
\hline SD ROI 1 contrast & 10.364 & 4.081 & 5 & 20 & $5.000-20.000$ & 0.0547 \\
\hline SD ROI 2 basal & 11.818 & 4.3547 & 4 & 17 & $4.000-17.000$ & 0.6073 \\
\hline SD ROI 2 contrast & 17.091 & 9.7719 & 5 & 34 & $5.000-34.000$ & 0.6494 \\
\hline SD ROI 3 basal & 7.909 & 2.0226 & 5 & 11 & $5.000-11.000$ & 0.3265 \\
\hline SD ROI 3 contrast & 10.091 & 4.6574 & 4 & 18 & $4.000-18.000$ & 0.6571 \\
\hline SD ROI 4 basal & 10.455 & 4.5905 & 5 & 20 & $5.000-20.000$ & 0.5176 \\
\hline SD ROI 4 contrast & 15.545 & 6.1703 & 9 & 25 & $9.000-25.000$ & 0.157 \\
\hline
\end{tabular}

lipid components close to the intima layer and it is this part of the plaque that may evolve to rupture and embolization.

By comparing the attenuation values among the four different ROIs the Mann-Whitney test did not show statistical differences among the four ROIs in the basal scan, whereas in the scan performed after contrast material administration in most cases there were difference among ROI values. This is an interesting point because shows 2 different types of information: 1) in the basal scan there are some differences (Table 1) but these are not statistically significant 2) After contrast material the differences between the ROI are statistically significant.

Table 2 Mann-Whitney analysis of the ROI attenuation values ( $p$ values are given)

Basal Attenuation values

\begin{tabular}{|c|c|c|c|c|}
\hline & $\mathrm{ROI} 1$ & $\mathrm{ROI} 2$ & $\mathrm{ROI} 3$ & $\mathrm{ROI} 4$ \\
\hline ROI 1 & $x$ & 0,1006 & 0,2122 & 0,078 \\
\hline $\mathrm{ROI} 2$ & & $x$ & 0,695 & 0,524 \\
\hline $\mathrm{ROI} 3$ & & & $x$ & 0,722 \\
\hline $\mathrm{ROI} 4$ & & & & $x$ \\
\hline \multicolumn{5}{|c|}{ Post contrast Attenuation values } \\
\hline & ROI 1 & ROI 2 & $\mathrm{ROI} 3$ & $\mathrm{ROI} 4$ \\
\hline ROI 1 & $x$ & $0,0086^{*}$ & $0,0053^{*}$ & $0,0008^{*}$ \\
\hline $\mathrm{ROI} 2$ & & $x$ & 0,1311 & $0,0488^{*}$ \\
\hline $\mathrm{ROI} 3$ & & & $x$ & $0,0115^{*}$ \\
\hline $\mathrm{ROI} 4$ & & & & $x$ \\
\hline
\end{tabular}

*statistically significant difference
These results indicate that ROI sampling for the $\mathrm{HU}$ assessment of the carotid artery plaque in the scan acquired after administration of contrast material can produce heterogeneous results according to the position of the ROI in the plaque and that the values are statistically different in the different part of the plaque. Moreover this heterogeneity is mainly due to the effect of the contrast material, as demonstrated by the fact that in the basal scan these differences are not significant whereas after contrast medium become statistically significant. Therefore, the difference of attenuation identified in the ROI may represent a difference of contrast material

Table 3 Mann-Whitney analysis of the Standard Deviation of $\mathrm{ROI}$ attenuation values ( $p$ values are given)

Basal Attenuation values

$\begin{array}{lllll} & \text { ROI 1 } & \text { ROI 2 } & \text { ROI 3 } & \text { ROI 4 } \\ \text { ROI 1 } & x & 0,0196 & 0,691 & 0,1062 \\ \text { ROI 2 } & & x & 0,0351 & 0,3579 \\ \text { ROI 3 } & & & x & 0,1779 \\ \text { ROI 4 } & & & & x\end{array}$

Post contrast Attenuation values

\begin{tabular}{lllll} 
& ROl 1 & ROI 2 & ROI 3 & ROl 4 \\
ROI 1 & $x$ & 0,1306 & 0,818 & 0,0562 \\
ROI 2 & & $x$ & $0,071^{*}$ & 0,9215 \\
ROI 3 & & $x$ & 0,0417 \\
ROI 4 & & & $x$ \\
\hline *statistically significant differnce & & &
\end{tabular}

*statistically significant difference 


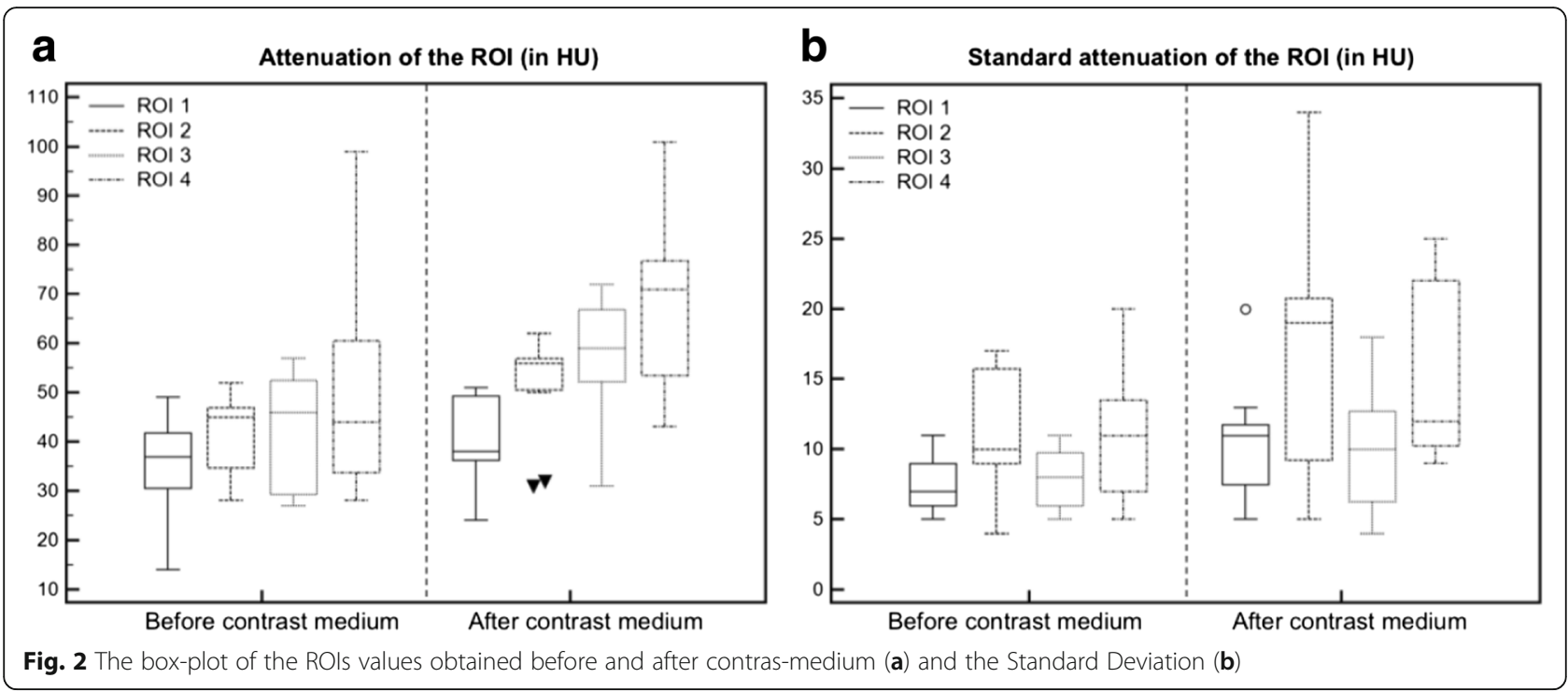

acquisition in different areas of the plaque. These results are in line with previously published papers that have demonstrated how carotid artery plaques shows enhancement after administration of contrast material [26-29]. Therefore, the attenuation value of the carotid artery plaque after administration of contrast material seems to represent not only the plaque type but also the amount of contrast material that enters in some areas of the plaques that is mainly due to the neovascularization or to the rupture of the fibrous cap $[9,30]$. This approach suggests that for the HU quantification of the tissue type the only pre-contrast $\mathrm{CT}$ should be performed and not the one after contrast material. This finding is concordant with a the study results of a paper published by Park et al [12] in 2012 where the authors demonstrated in a small cohort of carotid arteries $(n=$ 43) that is the attenuation value obtained in he precontrast scan that is associated with the cerebrovascular symptoms whereas the attenuation values obtained after contrast medium do not show statistically significant association with the cerebrovascular symptoms. Further analysis are needed to assess if the basal scan may be helpful to stratify the risk to develop cerebrovascular events.

We also assessed, using the Wilcoxon analysis, the global variation of attenuation of the plaque's ROI before and after contrast medium (basal value $=41.591 \mathrm{HU}$; contrast value $=54.159)$ and we found a statistically significant difference $(p$ value $=0.001)$.

\section{Conclusion}

In conclusion, results of our study suggest that the ROI sampling performed in the $\mathrm{CT}$ dataset acquired after administration of contrast medium show significant degree of heterogeneity and that the ROI have a statistically significant differences. This effect may be driven by a different amount of contrast acquisition in some areas of the carotid artery plaque.

\section{Abbreviations}

CT: Computed tomography; CTA: Computed tomography angiography; HU: Hounsfield units; MRI: Magnetic resonance imaging; ROI: Region of interest; US: Ultrasound

\section{Acknowledgements}

None.

\section{Funding}

None.

Availability of data and materials

For the present study electronical medical charts and imaging were used. No additional data collection was performed and institutional review board was not needed.

\section{Authors' contributions}

LS Manuscript ideation; data analysis; data collection; manuscript contribution and revision. MA Data analysis; manuscript contribution and revision. CDC Manuscript ideation; manuscript contribution and revision. MP Data analysis; data collection; manuscript contribution and revision. AB Data analysis; data collection; manuscript contribution and revision. RS Manuscript ideation; manuscript contribution and revision. MF Manuscript ideation; data analysis. AM Manuscript ideation; manuscript contribution and revision. PL Manuscript ideation; manuscript contribution and revision. RM Manuscript ideation; manuscript contribution and revision. JSS data analysis; manuscript contribution and revision. MW data analysis; manuscript contribution and revision. All authors read and approved the final manuscript.

\section{Competing interests}

The authors declare that they have no competing interests.

\section{Ethics approval and consent to participate}

The Institutional Review board of the Department of Radiology of the AOU of Cagliari (IR434) approved this retrospective study.

\section{Author details}

'Department of Radiology, Azienda Ospedaliero Universitaria (A.O.U.), di Cagliari - Polo di Monserrato, s.s. 554 Monserrato, Cagliari 09045, Italy. ${ }^{2}$ Department of Radiological, Oncological and Pathological Sciences, Sapienza University of Rome, Rome, Italy. ${ }^{3}$ Department of Radiological 
Sciences, Oncology and Pathology, University of Rome Sapienza-Polo Pontino, Latina, Italy. ${ }^{4}$ Department of Vascular Surgery, Azienda Ospedaliero Universitaria (A.O.U.), di Cagliari - Polo di Monserrato, s.s. 554 Monserrato, Cagliari 09045, Italy. ${ }^{5}$ Stroke and Monitoring Division, AtheroPoint, Roseville, CA, USA. ${ }^{6}$ Department of Radiology, Neuroradiology Section, Stanford University, Stanford, California, USA.

Received: 10 August 2016 Accepted: 28 October 2016

\section{Published online: 15 November 2016}

\section{References}

1. Go AS, Mozaffarian D, Roger VL, Benjamin EJ, Berry JD, Borden WB, Bravata DM, Dai S, Ford ES, Fox CS, Franco S, Fullerton HJ, Gillespie C, Hailpern SM, Heit JA, Howard VJ, Huffman MD, Kissela BM, Kittner SJ, Lackland DT, Lichtman JH, Lisabeth LD, Magid D, Marcus GM, Marelli A, Matchar DB, McGuire DK, Mohler ER, Moy CS, Mussolino ME, Nichol G, Paynter NP, Schreiner PJ, Sorlie PD, Stein J, Turan TN, Virani SS, Wong ND, Woo D, Turner MB, American Heart Association Statistics Committee and Stroke Statistics Subcommittee. Heart disease and stroke statistics-2013 update: a report from the American Heart Association. Circulation. 2013;127(1):e6-245.

2. Roger VL, Go AS, Lloyd-Jones DM, Benjamin EJ, Berry JD, Borden WB, Bravata DM, Dai S, Ford ES, Fox CS, Fullerton HJ, Gillespie C, Hailpern SM, Heit JA, Howard VJ, Kissela BM, Kittner SJ, Lackland DT, Lichtman JH, Lisabeth LD, Makuc DM, Marcus GM, Marelli A, Matchar DB, Moy CS, Mozaffarian D, Mussolino ME, Nichol G, Paynter NP, Soliman EZ, Sorlie PD, Sotoodehnia N, Turan TN, Virani SS, Wong ND, Woo D, Turner MB, American Heart Association Statistics Committee and Stroke Statistics Subcommittee. Executive summary: heart disease and stroke statistics-2012 update: a report from the American Heart Association. Circulation. 2012;125(1):188-97.

3. Imray $\mathrm{CH}$, Tiivas $\mathrm{CA}$. Are some strokes preventable? The potential role of transcranial doppler in transient ischaemic attacks of carotid origin. Lancet Neurol. 2005;4(9):580-6.

4. Rothwell PM, Warlow CP. Prediction of benefit from carotid endarterectomy in individual patients: a risk-modelling study. European Carotid Surgery Trialists' Collaborative Group. Lancet. 1999;353(9170):2105-10.

5. Alamowitch S, Eliasziw M, Algra A, Meldrum H, Barnett HJ, North American Symptomatic Carotid Endarterectomy Trial (NASCET) Group. Risk, causes, and prevention of ischaemic stroke in elderly patients with symptomatic internal-carotid-artery stenosis. Lancet. 2001;357(9263):1154-60.

6. Parmar JP, Rogers WJ, Mugler JP 3rd, Baskurt E, Altes TA, Nandalur KR, Stukenborg GJ, Phillips CD, Hagspiel KD, Matsumoto AH, Dake MD, Kramer CM. Magnetic resonance imaging of carotid atherosclerotic plaque in clinically suspected acute transient ischemic attack and acute ischemic stroke. Circulation. 2010;122(20):2031-8.

7. Hellings WE, Peeters W, Moll FL, Piers SR, van Setten J, Van der Spek PJ, de Vries JP, Seldenrijk KA, De Bruin PC, Vink A, Velema E, de Kleijn DP, Pasterkamp G. Composition of carotid atherosclerotic plaque is associated with cardiovascular outcome: a prognostic study. Circulation. 2010;121(17):1941-50.

8. Zainon R, Ronaldson JP, Janmale T, Scott NJ, Buckenham TM, Butler AP, Butler PH, Doesburg RM, Gieseg SP, Roake JA, Anderson NG. Spectral CT of carotid atherosclerotic plaque: comparison with histology. Eur Radiol. 2012;22(12):2581-8.

9. Saba L, Tamponi E, Raz E, Lai L, Montisci R, Piga M, Faa G. Correlation between fissured fibrous cap and contrast enhancement: preliminary results with the use of CTA and histologic validation. AJNR Am J Neuroradiol. 2013; 24 [Epub ahead of print].

10. Park JK, Sung YH, Jeong SY, Lee JH. Higher precontrast CT density of the carotid plaque in the symptomatic patients. Eur J Radiol. 2012;81(9):2386-8.

11. Saba L, Raz E, Grassi R, Di Paolo PL, lacomino A, Montisci R, Piga M. Association between the volume of carotid artery plaque and its subcomponents and the volume of white matter lesions in patients selected for endarterectomy. AJR Am J Roentgenol. 2013;201(5):W747-52.

12. Dean N, Lari H, Saqqur M, Amir N, Khan K, Mouradian M, Salam A, Romanchuk H, Shuaib A. Reliability of carotid doppler performed in a dedicated stroke prevention clinic. Can J Neurol Sci. 2005;32(3):327-31.

13. Singh N, Moody AR, Rochon-Terry G, Kiss A, Zavodni A. Identifying a high risk cardiovascular phenotype by carotid MRI-depicted intraplaque hemorrhage. Int J Cardiovasc Imaging. 2013;29(7):1477-83.

14. Saba L, Caddeo G, Sanfilippo R, Montisci R, Mallarini G. CT and ultrasound in the study of ulcerated carotid plaque compared with surgical results: potentialities and advantages of multidetector row $\mathrm{CT}$ angiography. AJNR Am J Neuroradiol. 2007;28(6):1061-6.

15. Chu B, Yuan C, Takaya N, Shewchuk JR, Clowes AW, Hatsukami TS. Images in cardiovascular medicine. Serial high-spatial-resolution, multisequence magnetic resonance imaging studies identify fibrous cap rupture and penetrating ulcer into carotid atherosclerotic plaque. Circulation. 2006;113(12):e660-1.

16. Kampschulte A, Ferguson MS, Kerwin WS, Polissar NL, Chu B, Saam T, Hatsukami TS, Yuan C. Differentiation of intraplaque versus juxtaluminal hemorrhage/thrombus in advanced human carotid atherosclerotic lesions by in vivo magnetic resonance imaging. Circulation. 2004;110(20):3239-44. E.

17. Saba L, Argiolas GM, Siotto P, Piga M. Carotid artery plaque characterization using CT multienergy imaging. AJNR Am J Neuroradiol. 2013;34(4):855-9.

18. Anzidei M, Napoli A, Zaccagna F, Di Paolo P, Saba L, Cavallo Marincola B, Zini C, Cartocci G, Di Mare L, Catalano C, Passariello R. Diagnostic accuracy of colour Doppler ultrasonography, CT angiography and blood-poolenhanced MR angiography in assessing carotid stenosis: a comparative study with DSA in 170 patients. Radiol Med. 2012;117(1):54-71.

19. Saba L, Anzidei M, Marincola BC, Piga M, Raz E, Bassareo PP, Napoli A, Mannelli L, Catalano C, Wintermark M. Imaging of the carotid artery vulnerable plaque. Cardiovasc Intervent Radiol. 2013. [Epub ahead of print], PubMed PMID: 23912494.

20. de Weert TT, de Monyé C, Meijering E, Booij R, Niessen WJ, Dippel DW, van der Lugt A. Assessment of atherosclerotic carotid plaque volume with multidetector computed tomography angiography. Int J Cardiovasc Imaging. 2008;24(7):751-9. doi:10.1007/s10554-008-9309-1.

21. Saba L, Mallarin G. Window settings for the study of calcified carotid plaques with multidetector CT angiography. AJNR Am J Neuroradiol. 2009;30(7):1445-50.

22. Nandalur KR, Baskurt E, Hagspiel KD, Phillips CD, Kramer CM. Calcified carotid atherosclerotic plaque is associated less with ischemic symptoms than is noncalcified plaque on MDCT. AJR Am J Roentgenol. 2005;184(1):295-8.

23. Saba L, Montisci R, Sanfilippo R, Mallarini G. Multidetector row CT of the brain and carotid artery: a correlative analysis. Clin Radiol. 2009;64(8):767-78.

24. Redgrave JN, Lovett JK, Gallagher PJ, Rothwell PM. Histological assessment of 526 symptomatic carotid plaques in relation to the nature and timing of ischemic symptoms: the Oxford plaque study. Circulation. 2006;113(19):2320-8.

25. Lovett JK, Redgrave JN, Rothwell PM. A critical appraisal of the performance, reporting, and interpretation of studies comparing carotid plaque imaging with histology. Stroke. 2005;36(5):1091-7.

26. Saba L, Piga M, Raz E, Farina D, Montisci R. Carotid artery plaque classification: does contrast enhancement play a significant role? AJNR Am J Neuroradiol. 2012;33(9):1814-7.

27. Saba L, Mallarini G. Carotid plaque enhancement and symptom correlations: an evaluation by using multidetector row CT angiography. AJNR Am J Neuroradiol. 2011;32(10):1919-25.

28. Horie N, Morikawa M, Ishizaka S, Takeshita T, So G, Hayashi K, Suyama K, Nagata I. Assessment of carotid plaque stability based on the dynamic enhancement pattern in plaque components with multidetector CT angiography. Stroke. 2012;43(2):393-8.

29. Romero JM, Babiarz LS, Forero NP, Murphy EK, Schaefer PW, Gonzalez RG, Lev MH. Arterial wall enhancement overlying carotid plaque on CT angiography correlates with symptoms in patients with high grade stenosis. Stroke. 2009:40(5):1894-6.

30. Saba L, Lai ML, Montisci R, Tamponi E, Sanfilippo R, Faa G, Piga M. Association between carotid plaque enhancement shown by multidetector CT angiography and histologically validated microvessel density. Eur Radiol. 2012;22(10):2237-45. 